\title{
Comparison of SETs in Bipolar Linear Circuits Generated with an Ion Microbeam, Laser Light and Circuit Simulation
}

\author{
Ron Pease, Senior Member, IEEE, Andrew Sternberg, Younes Boulghassoul, \\ Lloyd Massengill, Senior Member, IEEE, Stephen Buchner, Member, IEEE, Dale McMorrow, \\ Dave Walsh, Gerald Hash, Stephen LaLumondiere, Member, IEEE and Steven Moss, Senior Member, IEEE
}

\begin{abstract}
Generally good agreement was obtained between the single-event output voltage transient waveforms obtained by exposing individual circuit elements of a bipolar comparator and operational amplifier to an ion microbeam, a pulsed laser beam, and circuit simulations using SPICE. The agreement was achieved by adjusting the amounts of charge deposited by the laser or injected in the SPICE simulations. The implications for radiation hardness assurance are discussed.
\end{abstract}

\section{INTRODUCTION}

Single-Event Transients (SETs) were first reported in bipolar linear circuits in 1993 [1]. Since that time many part types have been characterized for SETs in a variety of circuit configurations and with a wide range of bias conditions $[2,3]$. Bipolar linear circuit SETs have been shown to be responsible for system upsets [3] and, hence, have become an issue for space system hardness assurance.

Last year a study was presented to determine the threshold critical charge for bipolar linear SETs [4]. That study involved testing individual circuit transistors with an ion microbeam and laser to determine the most sensitive transistors and the types of circuit output voltage transients produced. Also presented were the results of circuit simulations of the SETs by injecting current source transients in sensitive transistor junctions.

\footnotetext{
Manuscript received July 16, 2002

R.L. Pease is with RLP Research, Inc.,, Albuquerque, NM 87122 USA (Tel. 505 -856-1199, e-mail: rpease@ mrcmicroe.com).

A.Sternberg, is with the Department of Electrical Engineering, Vanderbilt University, Nashville TN 37235 USA (Tel. 615-343-6705 andrew.l.sternberg@vanderbilt.edu).

Y.Boulghassoul; is with the Department of Electrical Engineering, Vanderbilt University, Nashville TN 37235 (Tel. 615-343-6704, e-mail; younes.boulghassoul@vandebilt.edu;

L. Massengill is with the Department of Electrical Engineering, Vanderbilt Univ. Nashville, TN 37235 USA (Tel: 615-343-6677, e-mail; lloyd.massengill@vanderbilt.edu).

S. Buchner is with QSS Group Inc. Seabrook MD 20706 USA (Tel. 301286-5019, email: sbuchner@ pop500.gsfc.nasa.gov)

D. McMorrow is with the Naval Research Laboratory, Washington DC 20375 USA (Tel. 202-767-5469, e-mail mcmorrow@ ccs.nrl.navy.mil).

D. Walsh is with Sandia National Labs, Albuquerque NM 87185 USA (Tel. 505-8449590; e-mail dswalsh@ sandia.gov).

G. Hash is with Sandia National Labs., Albuquerque, NM 87185 USA (Tel. 505-845-8010, e-mail: hashgl@ sandia.gov).

S. LaLumondiere is with The Aerospace Corporation, Los Angeles CA 90009 USA (Tel. 310-336-6801 e-mail: stephen.lalumondiere@ aero.org).

S. Moss is with The Aerospace Corporation, Los Angeles, CA 90009 USA (Tel. 310-336-9216, e-mail; steven.moss@ @aero.org)
}

Detailed circuit transistor level studies, such as these, are part of a larger program to develop bipolar linear SET hardness assurance approaches with a goal of minimizing the amount of broad-beam heavy-ion testing that must be performed to guarantee that SETs in linear circuits will not cause system level failures. The approach involves compiling a canonical set of heavy-ion, pulsed-laser and circuitsimulation SET data for a particular device. If all the data are reasonably consistent, it should be possible to avoid additional heavy-ion testing of an identical device for those cases where its intended use is in a configuration or bias for which testing with heavy ions was not performed. Instead, the required data on SET pulse shape can be obtained through a combination of tests using a pulsed laser and circuit modeling. The usual long delays in obtaining SET data from an accelerator can thereby be avoided.

In this paper we compare the SET waveforms for the National Semiconductor (NSC) LM111 voltage comparator and the NSC LM124 quad operational amplifier for each of these three approaches. We find that, in all cases, there is fairly good agreement between the SET waveforms. In general, the pulsed-laser-induced waveforms tend to show better agreement with those generated by the ion-microprobe than do those from circuit-level simulations.

We also point out that the pulsed laser can assist in the development of accurate circuit models. Simulations require detailed information on transistor Gummel-Poon parameters that manufacturers are generally reluctant to divulge. The circuit modeler is forced to obtain the parameters either by reverse-engineering the part, or by making an "educated guess." In either case, the pulsed laser can play a significant role in validating the results, because the laser light can be focused on a known transistor junction and the resulting SET compared with that obtained for the same junction using circuit simulation. The circuit models for both the LM111 and the LM124 were developed first by reverse engineering the parts, and then by comparisons of the calculated and pulsed-laser induced SET waveforms. This approach is described in greater detail elsewhere [5,6].

Characterizing a circuit's SET response via circuit modeling involves a significant effort that must be repeated for each linear bipolar circuit with its own unique set of Gummel-Poon parameters. Such a large effort is unlikely to be cost effective for those devices with limited applications in space, and heavy-ion testing will likely be the approach of choice. However, for circuits that are used in a wide range of 
applications with different operating conditions, the use of limited heavy ion measurements together with pulsed laser measurements and accurate circuit modeling that can predict variations in SET characteristics with operating conditions, is extremely attractive. The results presented here are a first step towards achieving that goal.

\section{EXPERIMENTAL DESCRIPTION}

The Sandia microbeam facility uses a Tandem Van deGraaff Generator to accelerate the ion beam and magnetic focusing to achieve a spot size of about $1 \mu \mathrm{m}$ [7]. The maximum energy of the ions is about $50 \mathrm{MeV}$, but as a practical matter beams of $40 \mathrm{MeV}$ and below are normally used. In this study the ion beam was $40 \mathrm{MeV}$ chlorine, which has a range of about $11.5 \mu \mathrm{m}$ in silicon and an initial LET of about $18 \mathrm{MeV}-\mathrm{cm}^{2} / \mathrm{mg}$. For the circuits used in this study the overlayers are on the order of 3-4 $\mu \mathrm{m}$, which limits the penetration depth in the silicon to about 7.5-8.5 $\mu \mathrm{m}$, and the total deposited charge in the silicon to about $1.0 \mathrm{pC}$. Although the use of more deeply penetrating, higher LET ions is desirable, the values used in this study represent the limitations of the facility. The beam can be scanned in several modes. For this study the beam was either scanned horizontally or vertically or was rastered in a $x-y$ pattern. The flux was about 100 ions/second. In the $x-y$ scan the dwell time at each position was such that only one or two ions hit each "pixel".

The pulsed laser tests were performed using the NRL and Aerospace laser test facilities [8,9]. Although the NRL and Aerospace lasers have the same capabilities, they were operated under slightly different conditions. The NRL laser was operated at a wavelength of $590 \mathrm{~nm}$, a nominal pulse length of $1 \mathrm{ps}$, and a spot size of about $1.2 \mu \mathrm{m}$. Light with a wavelength of $590 \mathrm{~nm}$ has an absorption depth in silicon of approximately $2 \mu \mathrm{m}$. (The absorption depth is the depth at which the intensity of the incident light decreases to $1 / \mathrm{e}$ $(\sim 37 \%)$ of its value at the surface). The Aerospace laser was operated at $815 \mathrm{~nm}$, with a spot size of $1.5 \mu \mathrm{m}$ and an absorption depth of $11.5 \mu \mathrm{m}$. Limited experiments also were performed at $1260 \mathrm{~nm}$. The photon energy at $1260 \mathrm{~nm}$ is smaller than the Si bandgap, and absorption proceeds via a two-photon process [10]. The sub-bandgap laser has pulse duration of $120 \mathrm{fs}$ and a spot size of $1.6 \mu \mathrm{m}$. The absorption depends on the intensity [10]. Because the light is incident on the topside of the device for each of these experiments, junctions under metal cannot be reached directly. However, shielded junctions can be reached indirectly by irradiating as close as possible to the edge of the metal and relying on the diffusion of charge to the junction. Metal interference is not of significance for the LM124. Although the sensitive nodes of the LM111 are, in fact, covered with metal, no deleterious effect on the results was observed in the present experiments.

The bias conditions during irradiation for the LM111 and LM124 are given in a previous publication [4] and were as follows: 1) for the LM111 Vcc $= \pm 5 \mathrm{~V}$, the output load was $1.5 \mathrm{k} \Omega$ and the differential input voltage was $\pm 10 \mathrm{mV}$ and 2) for the LM124 Vcc $= \pm 6 \mathrm{~V}$, with an inverted gain of 20 and an input voltage of $-60 \mathrm{mV}$, giving an output voltage of $+1.2 \mathrm{~V}$.
For both experiments one sample of each type, with the lid removed, was irradiated. The SET experiments at the microbeam were performed in vacuum, and the laser experiments in air. The output transients were monitored on a digital oscilloscope (300 MHz bandwidth) using a FET probe attached to the circuit output through one foot of RG174 cable. The capacitance of the probe is $11 \mathrm{pF}$ and the bandwidth $1 \mathrm{GHz}$. The capacitance associated with the RG174 cable is $33 \mathrm{pF}$. Because the capacitance has an effect on some of the fast transients in the LM124, the identical setup was used for both ion microprobe and pulse laser experiments.

\section{CIRCUIT SIMULATIONS}

Circuit simulations were performed at Vanderbilt University using Silvaco SmartSPICE. Detailed circuit diagrams were extracted from the chip photomicrographs and SPICE models of the circuits were generated [5]. Transistor model parameters were derived from microprobe measurements of decoupled transistors of each major type (lateral and substrate pnp's and vertical npn's). Because of initial disagreements between the results of SPICE simulations and those of the pulsed laser, it was necessary to include parasitic elements, such as collector-to-substrate junctions and base spreading resistance in the SPICE model [6]. In addition, because some of the waveforms were very sensitive to output capacitance, all simulations included capacitors on the outputs equivalent to the cable and probe capacitances. Although simulations of the heavy-ion induced transient waveforms have been made for bipolar linear circuit transistors using the device physics code ATLAS, it was suggested that the circuit response was primarily a function of the total charge collected by the sensitive transistors and not the details of the injected current waveform [11]. Therefore, rather than use a mixed-mode simulator, the heavy ion strikes were simulated in SPICE using trapezoidal wave current pulses with $10 \mathrm{ps}$ rise and fall times. The amount of charge collected at a node was adjusted by varying the length and amplitude of the current pulse, while keeping it well below the microsecond response time of the circuit.

\section{RESULTS}

\section{A. $L M 111$.}

Figure 1 shows a photomicrograph of the LM111, and Fig. 2 a diagram of the input circuit. A more detailed discussion of the circuit is contained in last year's publication [4]. For the microbeam measurements, for each test condition $\left(\Delta \mathrm{V}_{\text {in }}= \pm 10 \mathrm{mV}\right)$, only one transistor on the die exhibits sensitivity to SETs. For a differential input voltage of +10 $\mathrm{mV}$ input transistor $\mathrm{Q} 1$ is sensitive; and for a differential input voltage of $-10 \mathrm{mV}$ input transistor Q2 is sensitive. Figure 3 shows the results of a $x-y$ scan of Q2 with the ion microbeam. Each highlighted pixel $(\sim 1 \mu \mathrm{m} \times 1 \mu \mathrm{m})$ represents a region that produced a SET at the LM111 output. The oscilloscope trigger was set so that only SETs with amplitudes greater than 0.2 volts were captured. The sensitive region of Q2 determined from the microbeam scan of fig. 3 is indicated by the striped region in Fig. 1. For this transistor, 
the entire sensitive region is located under a metal field plate over the base region of the transistor. This region is not accessible directly by the laser. However, by irradiating next to the field plate, as shown in Fig. 1, a SET response is obtained. Only three sides of the field-plate region of the base are sensitive to SET. The insensitive region of the base is the region next to the base contact that is not adjacent to the $\mathrm{p}+$ isolation region. The $\mathrm{p}+$ isolation region is connected to the substrate (collector region of this substrate pnp transistor). The simulations show that a collector-to-base or emitter-tobase strike is most sensitive.

Fig. 4 shows a comparison of the SET waveforms generated by irradiation of Q2 with the ion microbeam, the $590 \mathrm{~nm}$ laser, and SPICE simulation for $\Delta \mathrm{V}_{\text {in }}=-10 \mathrm{mV}$. To produce this and the following figures, the injected charge for the laser and the simulation are adjusted to best match the amplitude of the microbeam data. For the LM111 irradiated by $40 \mathrm{MeV} \mathrm{Cl}$ ions, only one type of SET waveform is observed for each sensitive region. However, the SET amplitudes vary according to the ion strike location, and all comparisons made in this paper are for SETs with maximum amplitude. As is evident in Fig. 4, the transients produced by the microbeam and laser are nearly identical, and the amount of charge deposited by each is similar (despite the metal coverage of the sensitive region). The simulation produces a slightly wider pulse that is missing the "undershoot" on the trailing edge, and the injected charge required to match the microbeam pulse is about a factor of 10 smaller than the charge deposited by the ion.

Fig. 5 shows a comparison of the SET waveforms for irradiation of LM111 transistor $\mathrm{Q} 1$ for $\Delta \mathrm{V}_{\text {in }}=+10 \mathrm{mV}$. Again, good agreement is observed between the microbeam, $590-\mathrm{nm}$ laser and simulated waveforms, but the charge required to generate the $0.2 \mathrm{~V}$ amplitude transient differ for the three cases. The reason for this discrepancy is not clear, but we note that for the ion and laser, the charge referred to in the figures is the deposited charge, whereas for the simulation the collected charge is the operative parameter. For $\Delta \mathrm{V}_{\mathrm{in}}=+10 \mathrm{mV}$, the region of $\mathrm{Q} 1$ that is sensitive in the microbeam and pulsed-laser tests is analogous to that observed for Q2 (for the output voltage low), except that the sensitive region is narrower. We note that for $40 \mathrm{MeV} \mathrm{Cl}$ ions the total charge deposited in the silicon is about $1.0 \mathrm{pC}$. Pulsed laser experiments at higher pulse energies indicate that other transistors of the LM111 (Q3, Q4, Q5a, and Q5b) become sensitive, and may contribute at higher LETs or higher total deposited charges [4].

\section{B. LM124.}

A photomicrograph of one of the four operational amplifiers on the LM124 die is shown in Fig. 6. The circuit components that produce SETs with the $40 \mathrm{MeV} \mathrm{Cl}$ ions are identified. A simplified circuit diagram is given in Fig. 7. The most sensitive region (by far) is the region labeled "R1" shown as a resistor from base to emitter of Q9. This "resistor" has been identified as a floating base transistor $[4,12]$. When this "resistor" is struck by an ion it turns "on", pulling the base of Q9 low and turning Q9 "off". This causes the output to go high, which is two diode drops below Vcc+ or $\sim 4.7 \mathrm{~V}$. The output voltage waveforms for irradiation of R1 and each of the nine transistors shown in Fig. 6 were recorded. Like the LM111, for $40 \mathrm{MeV} \mathrm{Cl}$ ions, only one SET waveform shape was observed for each sensitive region. Laser (590 $\mathrm{nm}$ ) tests and simulations using the same bias and output loading conditions were performed to determine the correlation to the SET waveforms generated by the heavy ions. The laser pulse energy and the collected charge in the simulated ion strike were adjusted for to best match the amplitude of the microbeam waveforms. The microbeam, laser and simulation transients for "R1" are shown in Fig. 8. The parameters used in generating the laser and simulations transients for R1 and each of the other transistors are collected in Table I.

The correlation between the laser and heavy ion waveforms for hits on R1 is quite good, as seen in the traces of Fig. 8. However, while the simulation shows reasonable qualitative correlation, the rise and fall times of the pulse are considerably longer than the experimental pulses. Fig. 9 shows the transients for Q2, Q3, Q4, Q5, and Q6. Q2, not shown in Fig. 7, is a substrate pnp with its base tied to the collector of Q3. In general, the charge required for the laser and simulations to match the ion SET waveform amplitude are slightly higher than the $1.0 \mathrm{pC}$ calculated as the charge deposited by the ion. For the data of Fig. 9, the laser- and ioninduced transients are nearly indistinguishable, and the simulated transients also exhibit good agreement with the experimental data. The values of deposited (laser) and collected (simulation) charge required to match the ion SET amplitudes are somewhat scattered, presumably for the reasons discussed above.

As illustrated in Fig. 10, the agreement between laser, ion and simulation for transistors Q9 and Q19 is less good. The origin of this generally poorer agreement is not clear at the present time.

For lateral pnp transistors Q18 and Q20 the SETs are of nearly equal amplitude but opposite polarity, with Q18 showing a positive transient and Q20 a negative transient. The waveforms for Q18 and Q20 are given in Fig. 11, again illustrating a good correlation between microbeam, laser and simulation. The microbeam pulse is somewhat narrower and has an undershoot that is not shown by the laser pulse and simulation.

Table 1 compares the amount of charge deposited by the laser light with the amount injected in the modeling approach to match the amplitude of the equivalent ion-generated SET. $1.0 \mathrm{pC}$ of charge was deposited for each of the ion-induced SETs. Quantitative differences between the amount of charge required by the optical pulses and simulations to match the ion-induced SET amplitude are evident. For the laser this may be attributed to the much shorter penetration depth (2 $\mu \mathrm{m})$ than that of the heavy ion $(7.5-8.5 \mu \mathrm{m})$, and the correspondingly higher pulse energy required to deposit sufficient charge at the deeper junctions. For the simulations the quantity of charge depends primarily on the transistor model parameters.

In previous studies, very good correlation between pulsedlaser and heavy-ion irradiation has been reported for SEU and SEL in a range of semiconductor technologies that are characterized by shallow junctions $[13,14]$. While the present study exhibits better than a factor of two agreement (in the 
deposited charge) between the ion and laser in all cases, the quantitative correlation is not, in general, of the same quality as in that previous work. This observation, we believe, is a consequence primarily of the complex, three-dimensional structures of the present bipolar devices, which have junctions as deep at $15 \mu \mathrm{m}$, and the differences in charge deposition profiles generated by the $40 \mathrm{MeV} \mathrm{Cl}$ ion and the $590 \mathrm{~nm}$ laser light. We note, however, that the transients reported here are specific to $40 \mathrm{MeV} \mathrm{Cl}$ ions, and that the amplitude, width, and shape of the measured transients are expected to vary with ion species, energy, and LET.

\section{DISCUSSION}

The test results shown here for the microbeam are somewhat limited because the energy of the ion limits the depth of penetration and the maximum charge that can be deposited in the sensitive volume. Hence in the case of the LM111 only one transistor was sensitive to the $40 \mathrm{MeV}$ chlorine ion for each test condition. Previous tests with the laser and circuit simulations have shown that several transistors on the LM111 are SET sensitive, as mentioned earlier [4]. However, although the results on the LM111 are limited, they do illustrate the degree of correlation that can be achieved with both the laser and with simulations. While the laser cannot directly penetrate the metal field plate of Q1 and Q2, it is capable of producing transients nearly identical to those produced by heavy ion irradiation by focusing on a region immediately adjacent to the sensitive region. This result apparently is due to the diffusion of charge under the metal. The waveforms for both the laser and simulation exhibit good correlation with that for the microbeam but, for the LM111, the total deposited charge required to match the ion induced SET is much less for the simulations. This result is not particularly surprising because of the distinction between deposited charge and collected charge, such that, in the absence of gain processes, the deposited charge should always be greater than the simulated (or collected) charge. The magnitude of the difference, however, suggests that the SPICE parameters for the LM111 transistor models require additional work. The results on the LM111 suggest that both the laser and simulation can be used to obtain fairly accurate SET pulse shapes for biases and configurations other than those tested with the microbeam.

For the case of the LM124, which is much more sensitive than the LM111, 10 transistors that are SET sensitive with the $40 \mathrm{MeV} \mathrm{Cl}$ microbeam. In most cases the waveforms generated with the ion microprobe agree well in both shape and pulse width (with adjustments in charge) with those generated by the laser and the simulations. For the most sensitive region on the LM124, the floating base transistor used as a "resistor", the simulation matched the shape of the waveform but had significantly longer rise and fall times, thus widening the SET pulse.

A primary limitation of the ion microbeam is the rather limited energy range over which micrometer resolution can be maintained. This translates into limitations on the total deposited charge and the penetration depth (range) of the ion. While the range of deposited charge for the microbeam test is somewhat limited, the deposited energy for the laser is almost unlimited. The results shown for the LM124 demonstrate that the laser and simulations can reproduce the SET transients produced by the $40 \mathrm{MeV} \mathrm{Cl}$ microbeam ions. However, the waveforms produced in a typical broad-beam experiment may include transients not observable in microbeam tests because of higher energies (and LETs) available at most heavy ion test facilities. In order to explore the waveforms generated by a sensitive transistor as a function of laser energy and penetration depth additional experiments were performed for a range of excitation conditions. The data of Fig. 12 illustrate the effects of variations in the deposited charge for LM124 transistors Q9 and Q19. In this series of experiments, the pulse energy of $590 \mathrm{~nm}$ laser pulses was varied by about a factor of 2 . The rather dramatic changes in the SET pulse properties are evident, with the rise time, amplitude, and shape of the undergoing significant evolution. The reason for this change in waveform is because the response of the LM124 depends on how hard the nodes are driven. Using the laser to characterize the SET response over a range of energies, provides a more complete indication of the SET response expected in both broad-beam heavy ion experiments, and in the natural space environment, both of which involve a wide range of higher energy and higher LET ions.

Additional experiments were performed on the lateral pnp transistor Q20 at 815-nm [9] and at sub-bandgap wavelengths $(1260 \mathrm{~nm})$ using the principle of two-photon absorption to generate carriers [10]. The 815-nm laser has a 1/e depth of $11.5 \mu \mathrm{m}$. At sub-bandgap wavelengths the location of maximum charge generation can be adjusted to any depth in the device. Figure 13 shows behavior of the SET induced on Q20 with $815 \mathrm{~nm}$ optical pulses as a function of the laser pulse energy (with is proportional to the quantity of deposited charge). These data illustrate that the polarity of the SET changes from negative, to bipolar, to positive as the pulse energy is increased. For sub-bandgap excitation similar behavior is observed as the location of the maximum charge deposition is moved (at constant pulse intensity) from the surface to a depth of about $9 \mu \mathrm{m}$ [10]. Similar behavior also is observed for 590-nm excitation as the pulse energy is varied. These results are consistent with a model in which Q20 contains two competing junctions at different depths below the surface, and suggest that the more sensitive junction is at the greater depth. At low laser pulse energies the amount of charge deposited near the top junction exceeds the SET threshold for that junction, whereas the amount of charge deposited at the lower junction is small due to the exponential decay of the light intensity with depth in the material [15]. As a result, the SETs are dominated by charge deposited near the top junction, giving rise to a negativegoing transient. At higher laser pulse energies more charge reaches the lower junction. The results of Fig. 13 indicate that the deeper junction is more sensitive and, at some critical quantity of charge, the response of this deeper junction dominates the response, resulting in SETs that are positive. This model is confirmed by two-photon measurements [10]. We note that the two-photon measurements also reveal a third, deeper junction that produces a SET of negative polarity [10]. The results of Figs. 12 and 13 illustrate that 
laser pulses with different characteristics can be powerful diagnostic tools that can be used to characterize the full range of SET waveforms that would be expected in a broad-beam test or in the radiation environment of space.

\section{CONCLUSION}

The results presented here demonstrate that generally good correlation between ion and laser induced SET waveforms can be achieved at the circuit component level. Furthermore, fair agreement also is obtained with the SET waveforms generated using SPICE circuit simulation. Differences in charge deposition and collection between the ion and laser are most likely due to differences in track structures and junction depths. These results suggest that it should be possible to minimize heavy ion testing by using laser irradiation together with simulation to explore system level SET response under a wide range of applications. In particular, it is clear from this study that both the pulsed laser and SPICE simulation approaches successfully bracket the range of pulse widths and pulse heights observed for the experimental conditions of this study, with quantitative agreement that should be adequate a hardness assurance program. To confirm the validity of this approach, in an extension of the present work, the current data set will be used to predict the SET responses of these circuits for previously untested configurations, and those predictions will be verified with heavy ion testing.

\section{ACKNOWLEDGMENTS}

This work was funded primarily by the Defense Threat Reduction Agency (DTRA) under contracts N00164-97-D0013 and DTRA01-00-C-0010 as part of the DTRA Radiation Hardened Microelectronics Program. The authors thank Lew Cohn of DTRA for his enthusiastic support. SPB wishes to thank NASA's Electronic Parts and Packaging Program for support. Work performed at The Aerospace Corporation was supported by U.S. Air Force Space and Missile Systems Center under Contract No. F04701-00-C0009 .

\section{REFERENCES}

[1] R. Koga, S. D. Pinkerton, S. C. Moss, D. C. Mayer, S. LaLumondiere, S. J. Hansel, K. B. Crawford and W. R. Crain, "Observation of Single Event Upsets in Analog Microcircuits", IEEE Trans. Nucl. Sci., vol. 40, 1838-1844, Dec. 1993.

[2] R. Ecoffet, R. S. Duzellier, P. Tastet, C. Aicardi, and M. Labrunee, "Observation of Heavy Ion Induced Transients in Linear Circuits", 1994 IEEE Radiation Effects Data Workshop Record, 72-77, 1994.

[3] R. Koga, S. H. Crain, K. B. Crawford, S. C. Moss, S. D. LaLumondiere and J. W. Howard, Jr., "Single Event Transient (SET) Sensitivity of Radiation Hardened and COTS Voltage Comparators", 2000 IEEE Radiation Effects Data Workshop Record, 53-60, 2000.

[4] R. L. Pease, A. Sternberg, L. Massengill, R. Schrimpf, S. Buchner, M. Savage, J. Titus, and T. Turflinger, "Critical Charge for Single-Event Transients in Bipolar Linear Circuits", IEEE Trans. Nucl. Sci., vol. 48, 19661972, Dec. 2001.

[5] Y. Boulghassoul, L. W. Massengill, A. L. Sternberg, R. L. Pease, S. P. Buchner, J. W. Howard, D. McMorrow, M. W. Savage, and C. Poivey, Circuit Modeling of the LM124 Operational Amplifier for Single-EventTransient Prediction", IEEE Trans. Nucl. Sci., vol. 49, Dec. 2002.
[6] A. L. Sternberg, L. W. Massengill, Y. Boulghassoul, S. P. Buchner, R. L. Pease, and M. W. Savage, "The Role of Parasitic Elements in the SingleEvent-Transient Response of Linear Circuits", IEEE Trans. Nucl. Sci., vol. 49, Dec. 2002.

[7] F. W. Sexton, "Microbeam Studies of Single Event Effects", IEEE Trans. Nucl. Sci., vol. 43, 687-695, Apr. 1996.

[8] J. S. Melinger, S. Buchner, D. McMorrow, W. J. Stapor, T. R. Weatherford and A. B. Campbell, "Critical Evaluation of the Pulsed Laser Method for Single Event Effects Testing and Fundamental Studies", IEEE Trans. Nucl. Sci., vol. 41, 2574-2584, Dec. 1994.

[9] S. D. LaLumondiere, S. C. Moss, R. Koga, and M. C. Maher. "LaserInduced and Heavy Ion-induced Single Event Transient(SET) Sensitivity Measurements on LM139 Comparators", IEEE Trans. Nucl. Sci., vol. 49, Dec. 2002

[10] D. McMorrow, W. T. Lotshaw, J. S. Melinger, S. P. Buchner, and R. L. Pease, "Sub-bandgap Laser Induced Single Event Effects: Carrier Generation via Two Photon Absorption", IEEE Trans. Nucl. Sci., vol. 49, Dec. 2002.

[11] P. Adell, R. D. Schrimpf, H. J. Barnaby, R. Marec, C. Charty, P. Calvel, C. Barillot and O. Mion, "Analysis of Single-Event Transients in Analog Circuits", IEEE Trans. Nucl. Sci., vol. 47, 2616-2623, Dec. 2000.

[12] H. Fred Barsun, NAVSEA Crane, unpublished work.

[13]. S.C. Moss, S.D. LaLumondiere, J.R. Scarpulla, K.P. MacWilliams, W.R. Crain, and R. Koga, "Correlation of Picosecond Laser-Induced Latchup and Energetic Particle-Induced Latchup in CMOS Test Structures," IEEE Trans. Nuc. Sci., vol. 42, 1948 Dec. 1995.

[14]. D. McMorrow, J.S. Melinger, S. Buchner, T. Scott, R.D. Brown, N.F. Haddad, "Application of a pulsed laser for evaluation and optimization of SEU-hard designs", IEEE Trans. Nuc. Sci. vol. 47, 559 Dec. 2000.

[15]. J.S. Melinger, D. McMorrow, A.B. Campbell, S. Buchner, L.H. Tran, A.R. Knudson, and W.R. Curtice, "Pulsed laser induced single event upset and charge collection measurements as a function of optical penetration depth," J. App. Phys., vol. 84, 6901998. 


\section{List of Figures and Tables}

TABLE I

CHARGE TO MATCH THE AMPLITUDES OF THE ION-INDUCED SETS. (IN EACH CASE, THE ION DEPOSITS 1.0 PC).

Figure 1. Photomicrograph of the LM111 input circuit showing sensitive regions of Q2 for microbeam and the location of the most sensitive spot for laser pulse injection.

Figure 2. Schematic diagram of LM111 input circuit.

Figure 3. X-Y scan of sensitive region of node Q2 of LM111 for microbeam irradiation illustrating the locations of ion strikes resulting in SET pulse of amplitude greater than $0.1 \mathrm{~V}$.

Figure 4. Comparison of microbeam, laser and simulation SET waveforms for $\Delta \mathrm{V}_{\text {in }}=-10 \mathrm{mV}$ for $\mathrm{Q} 2$ on LM111. Microbeam deposited charge: $1.0 \mathrm{pC}$; $590 \mathrm{~nm}$ laser: $1.2 \mathrm{pC}$; and simulation collected charge: $0.1 \mathrm{pC}$.

Figure 5. Comparison of microbeam, laser and simulation for SET waveform for $\Delta \mathrm{V}_{\mathrm{in}}=+10 \mathrm{mV}$ for Q1 on LM111. Microbeam deposited charge: $1.0 \mathrm{pC}$; $590 \mathrm{~nm}$ laser: $0.45 \mathrm{pC}$; and simulation collected charge: $0.15 \mathrm{pC}$

Figure 6. Photomicrograph of the LM124 operational amplifier showing SET sensitive components for the microbeam.

Figure 7. Simplified circuit diagram of LM124.

Figure 8. SET waveforms for microbeam, laser and simulation for hits on "R1".

Figure 9. SET waveforms for microbeam, laser and simulation for hits on Q2, Q3, Q4, Q5, and Q6.

Figure 10. SET waveforms for microbeam, laser and simulation for hits on Q9 and Q19.

Figure 11. SET waveforms for microbeam, laser and simulation for hits on Q18.

Figure 12. Pulse shape as a function of deposited energy for laser irradiations of Q9 and Q19.

Figure 13. Q20 SET waveform for different laser energies for $815 \mathrm{~nm}$ laser. 


\begin{tabular}{ccc}
\hline Transistor & $\begin{array}{c}\text { Simulated Charge } \\
(\mathrm{pC})\end{array}$ & $\begin{array}{c}\text { Laser Charge } \\
(\mathrm{pC})\end{array}$ \\
\hline Q2bc & 1.8 & 1.3 \\
Q3ce & 1.4 & 0.9 \\
Q4bc & 1.1 & 1.0 \\
Q5bc & 1.6 & 0.9 \\
Q6bc & 1.35 & 2.0 \\
Q9bc & 3.5 & 1.4 \\
Q18bc & 1.2 & 0.9 \\
Q19C5bc & 3.0 & 1.8 \\
Q20bc & 1.3 & 1.0 \\
"R1"bc & 1.0 & 0.5 \\
\hline
\end{tabular}




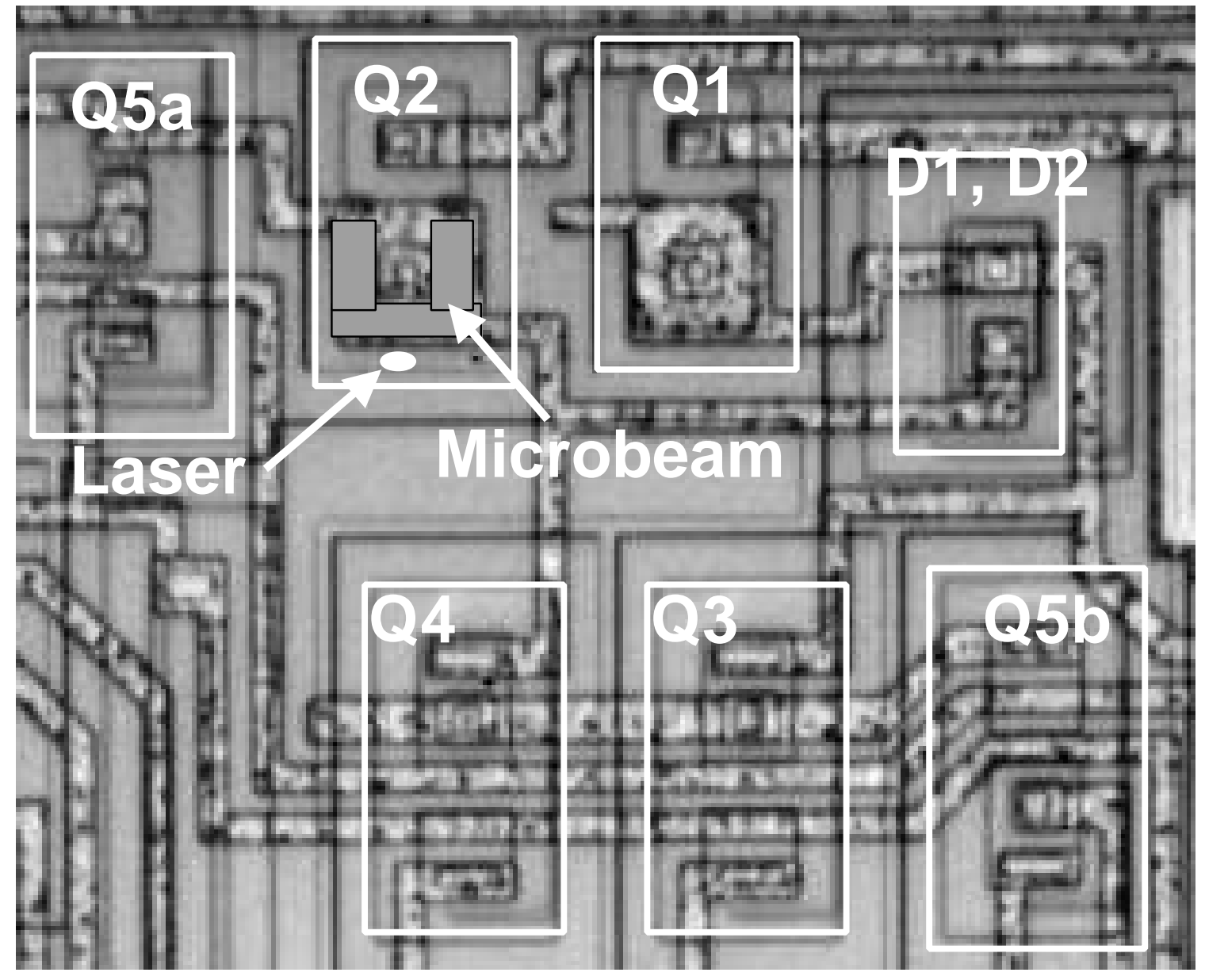




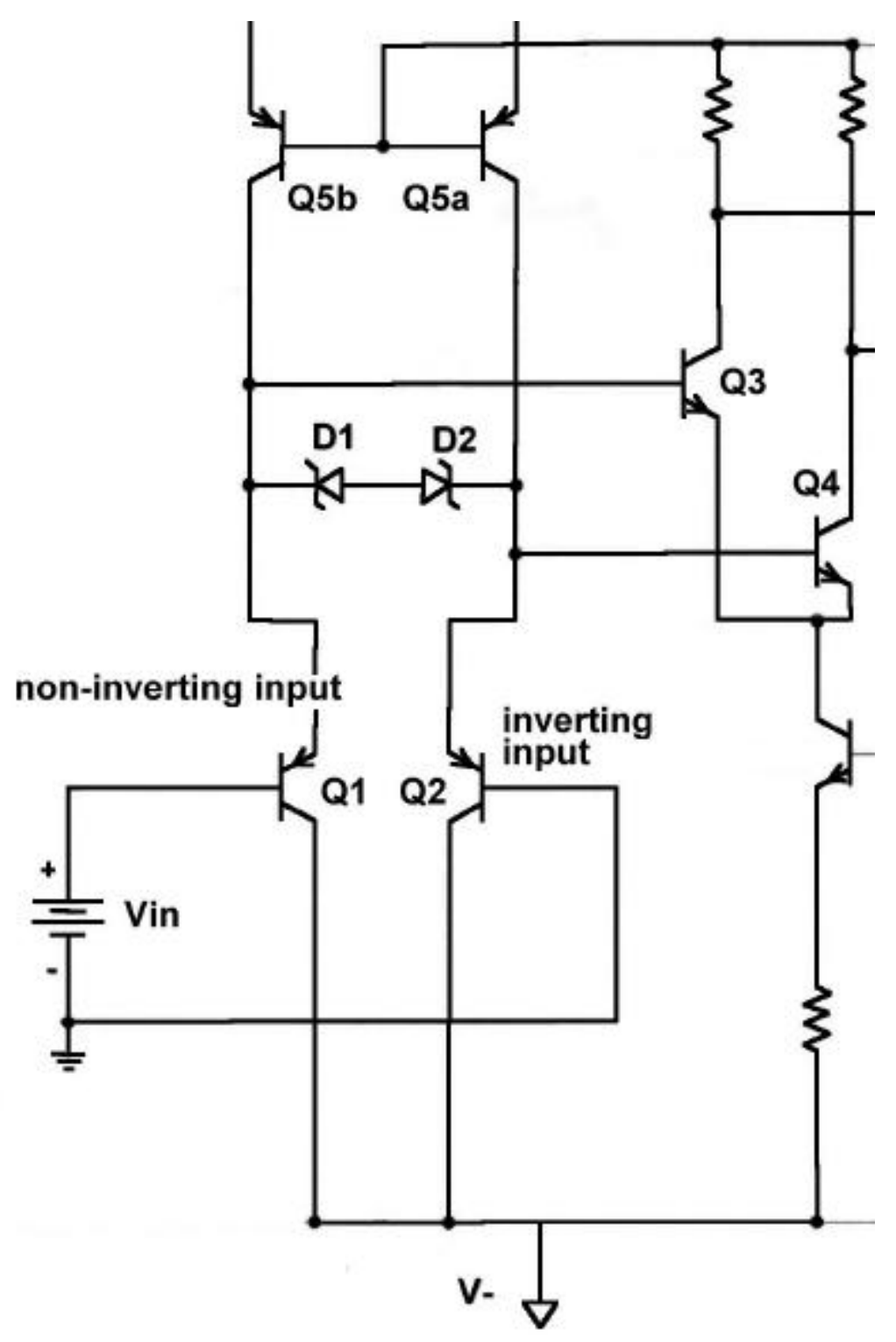




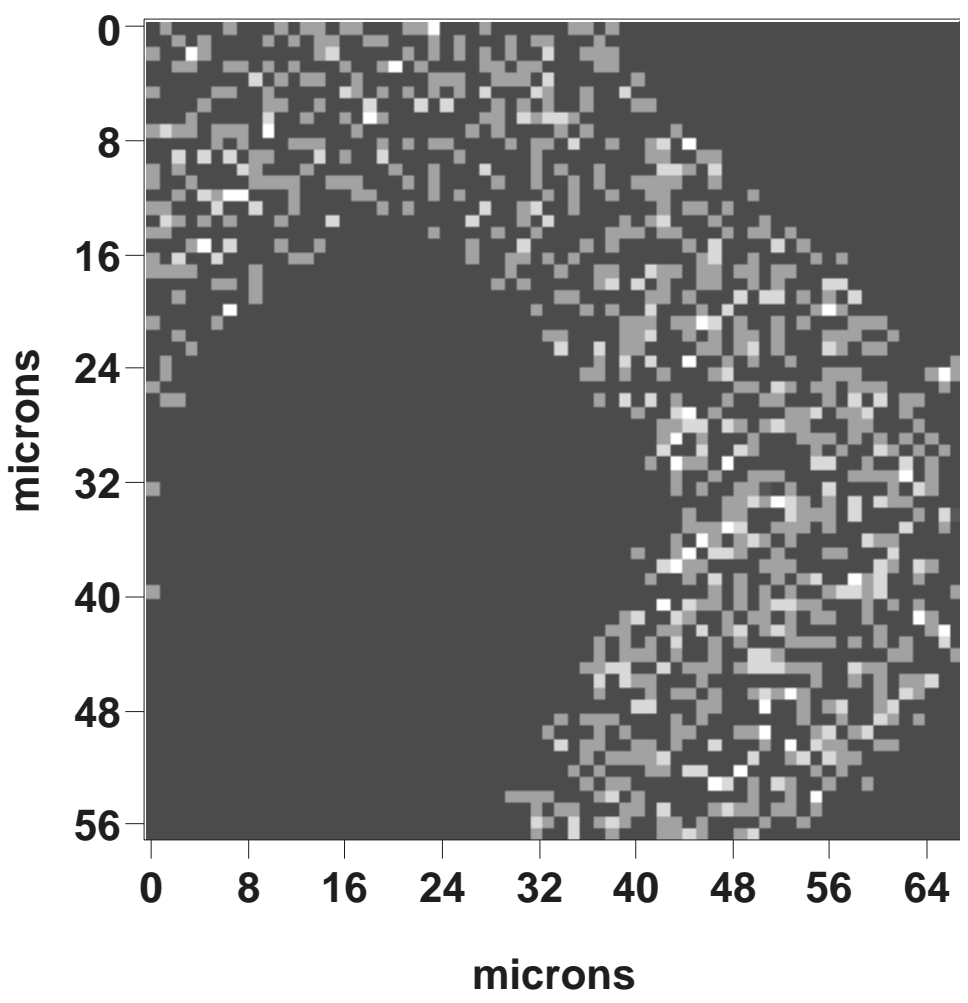




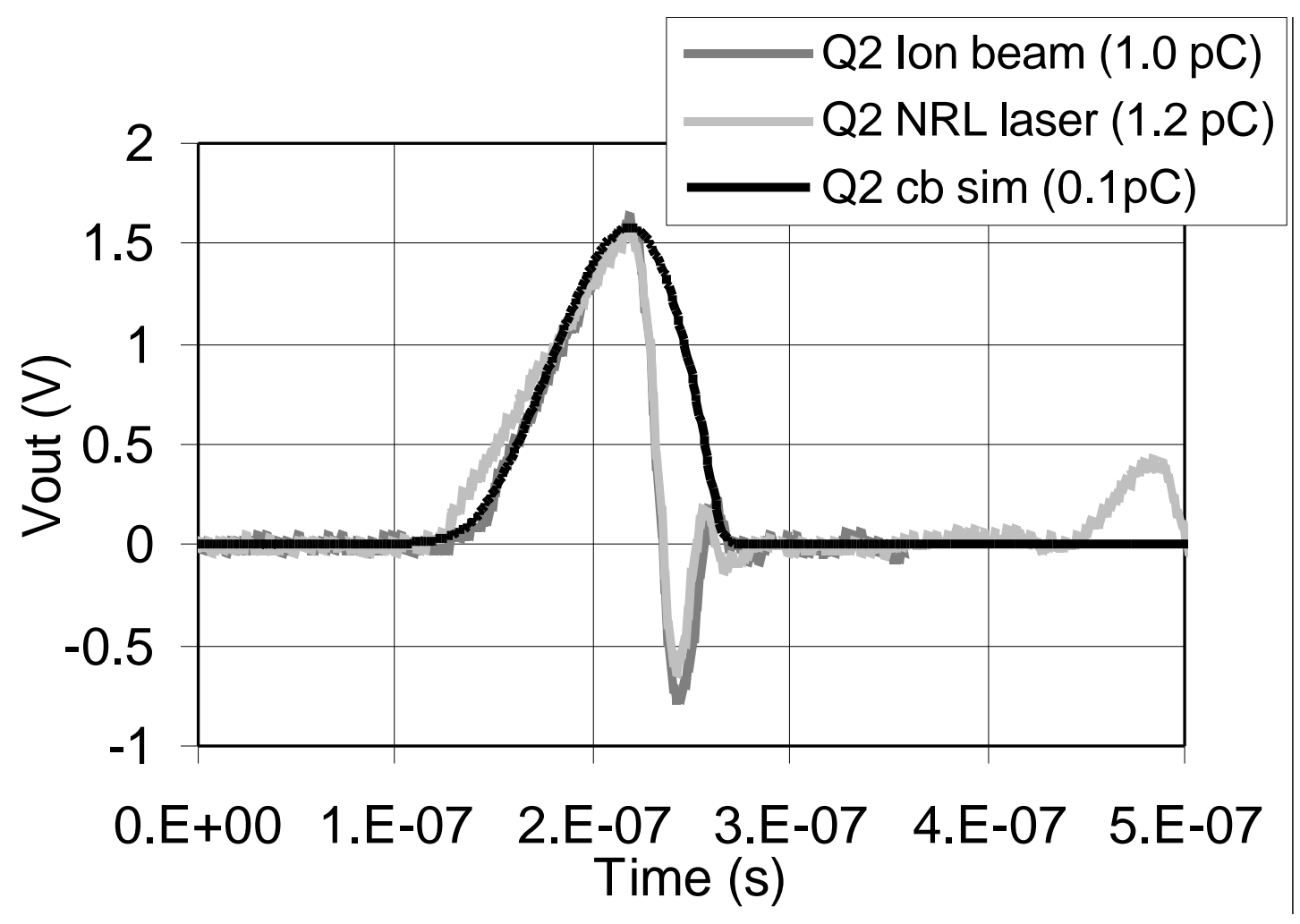




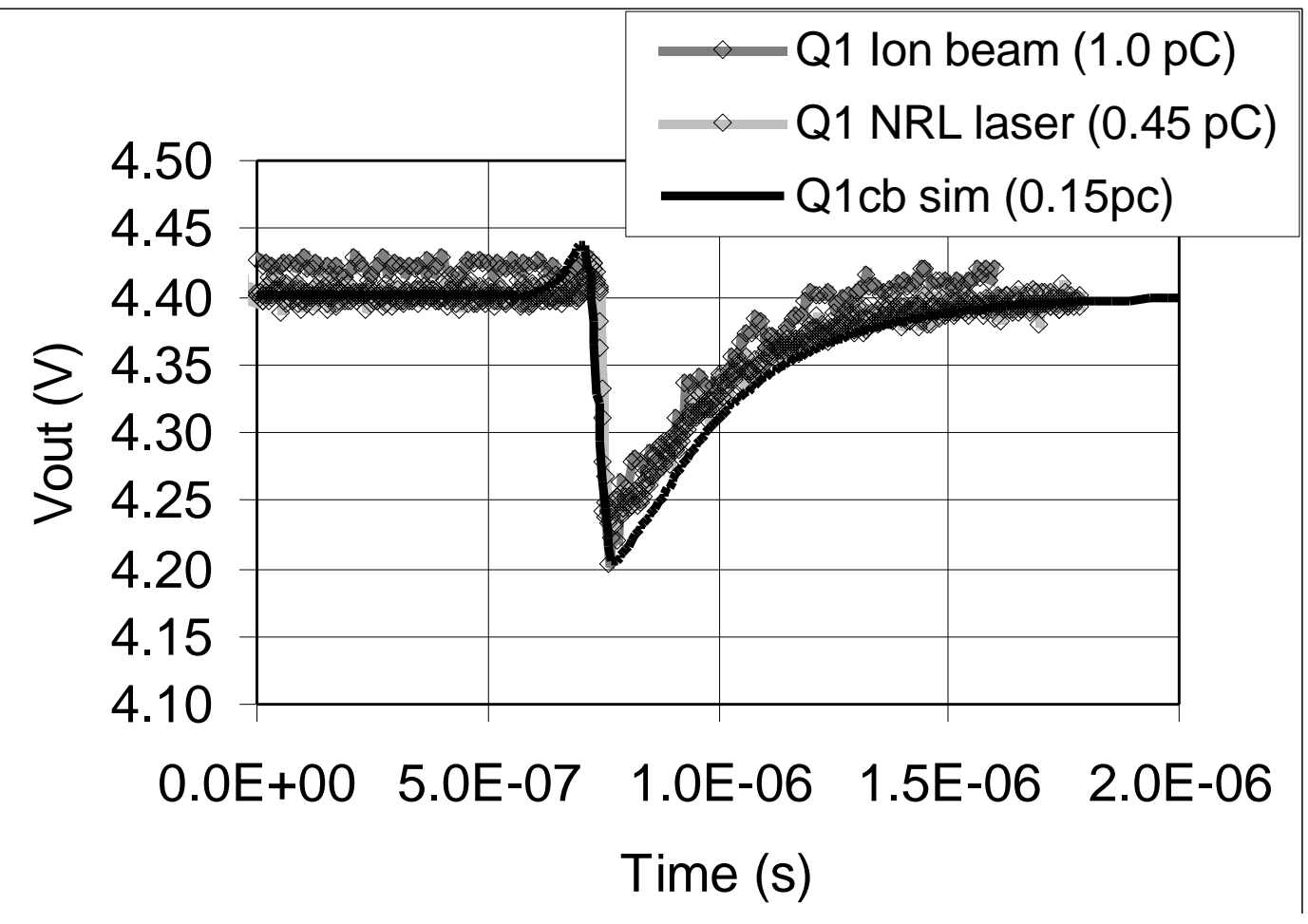




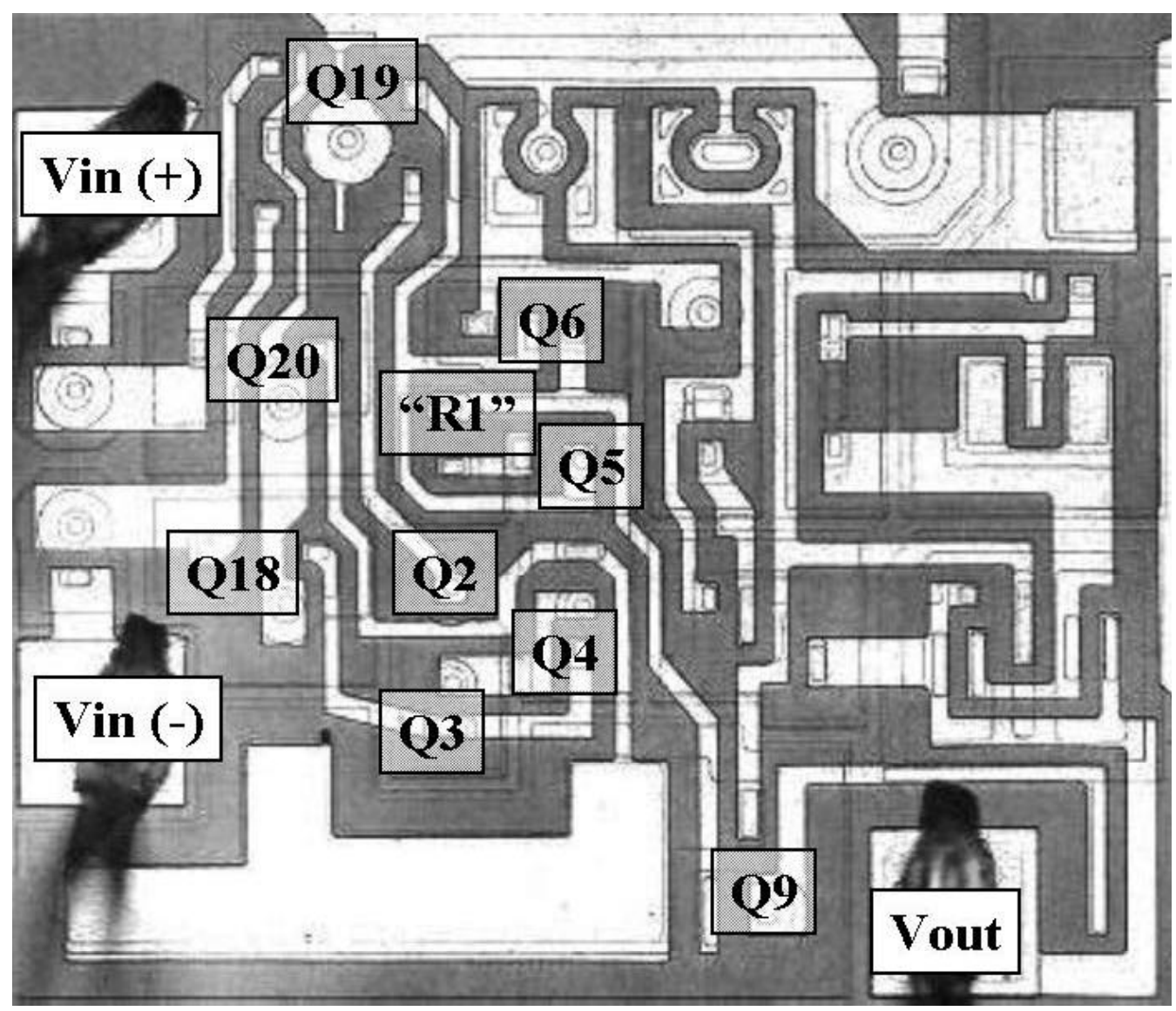




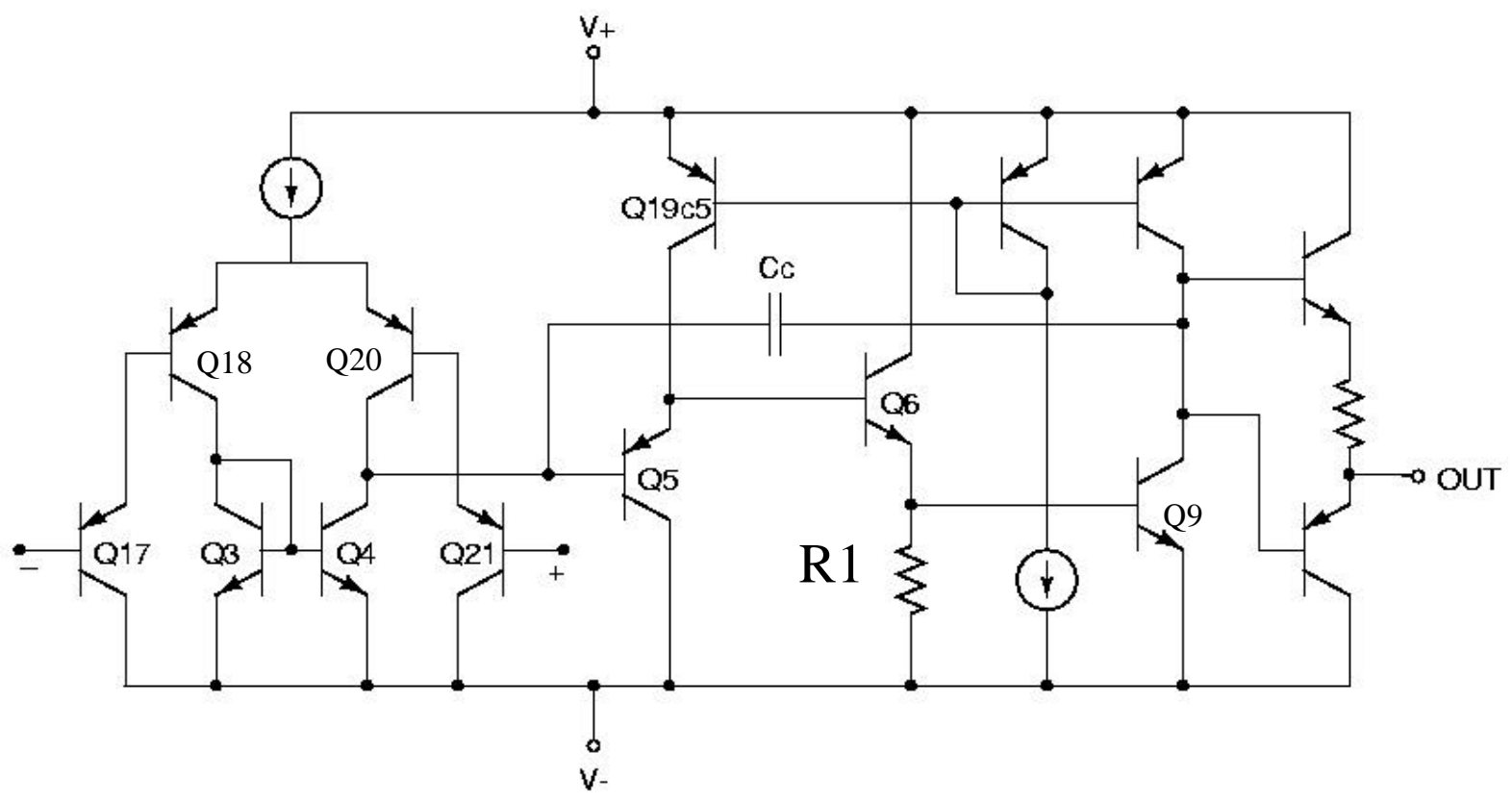




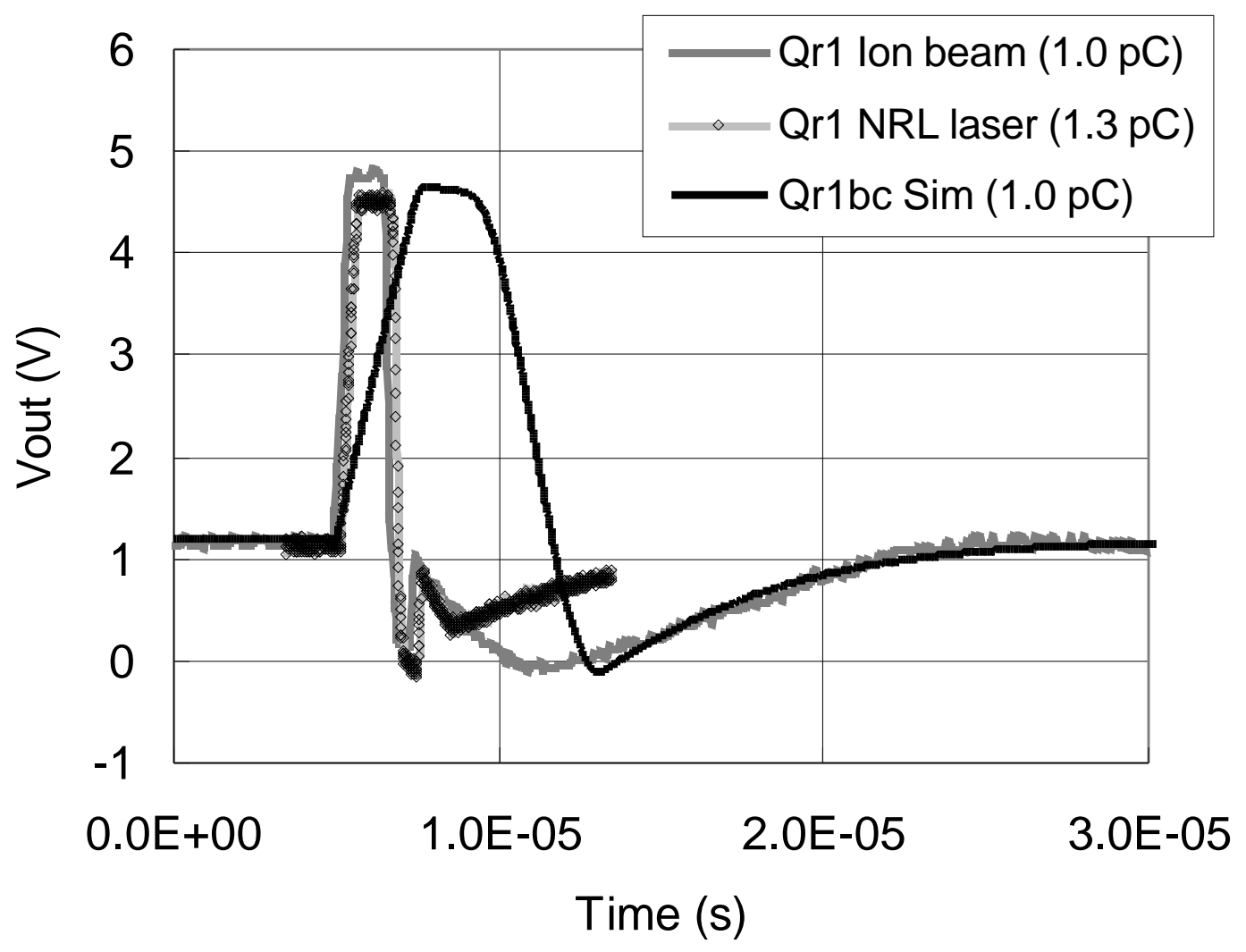




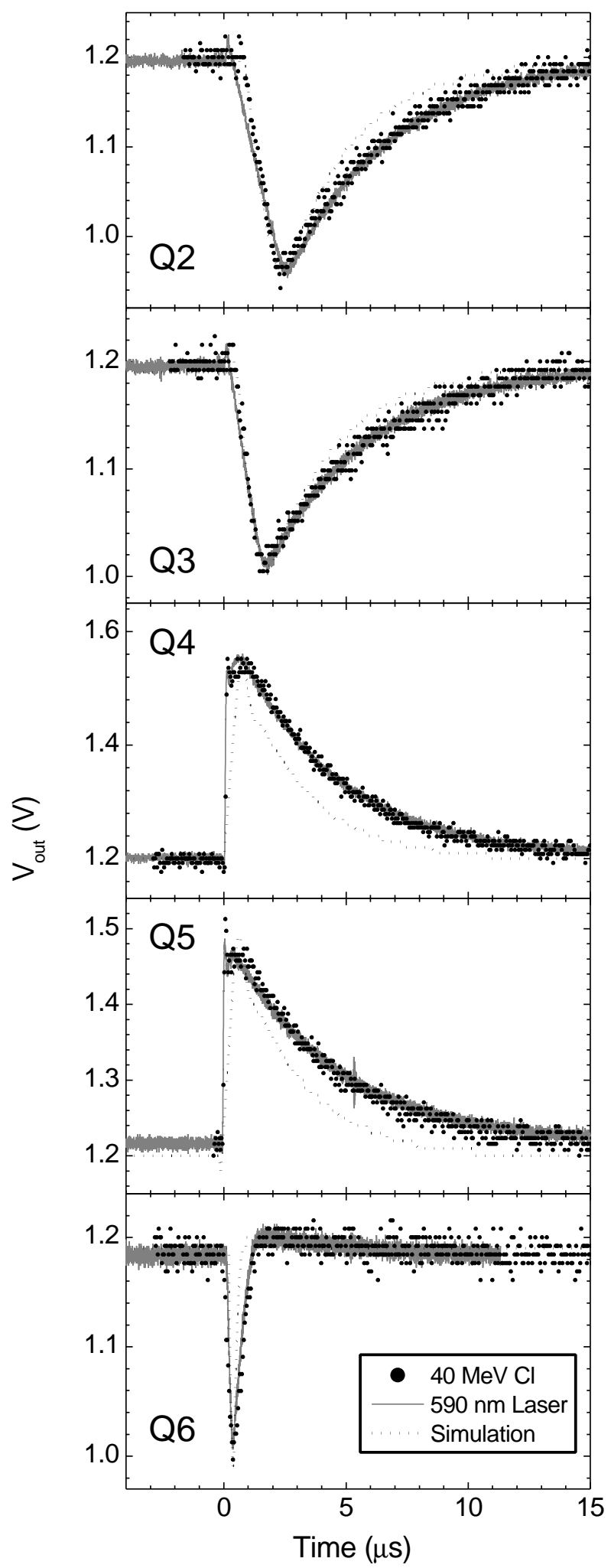



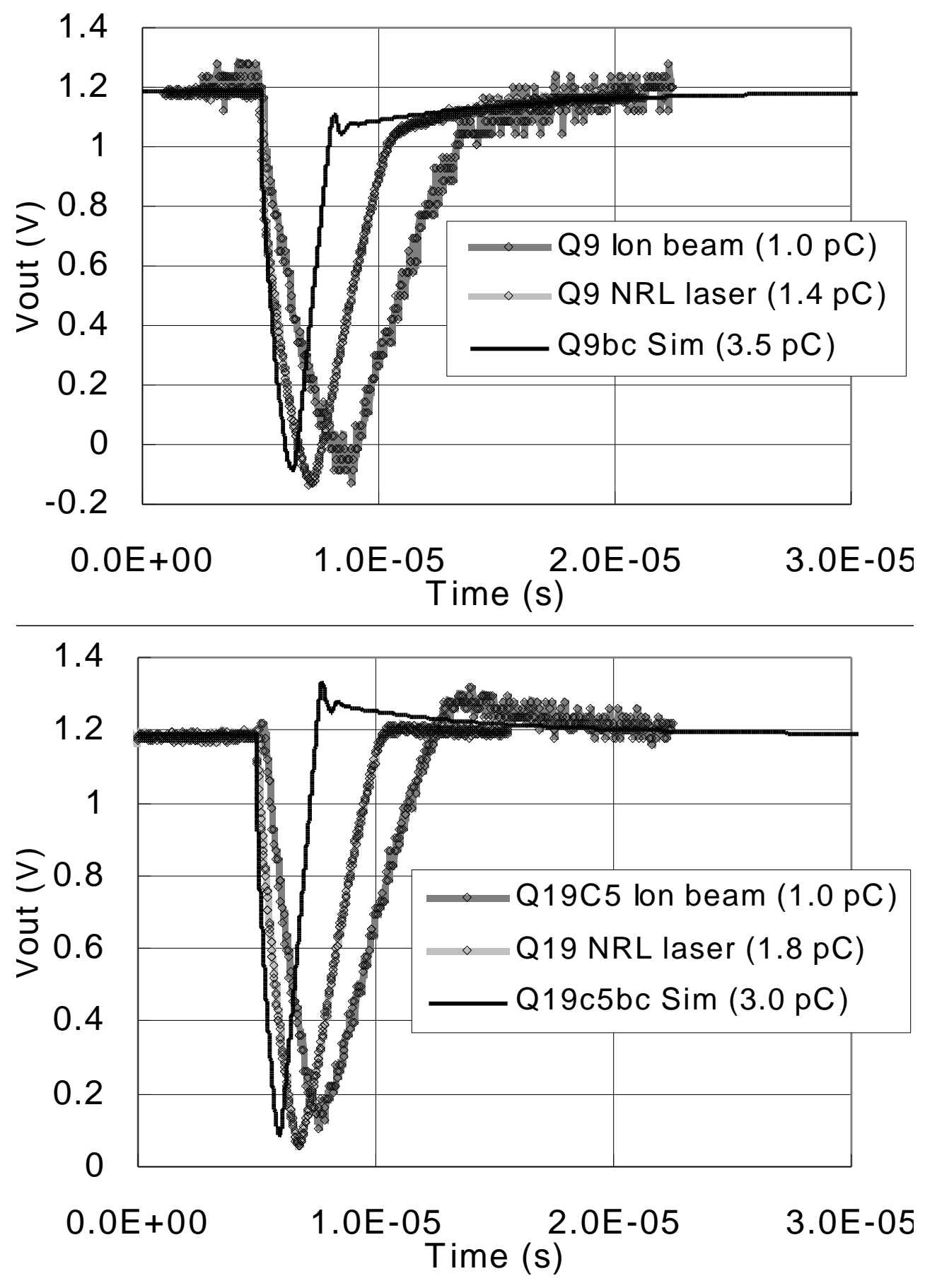


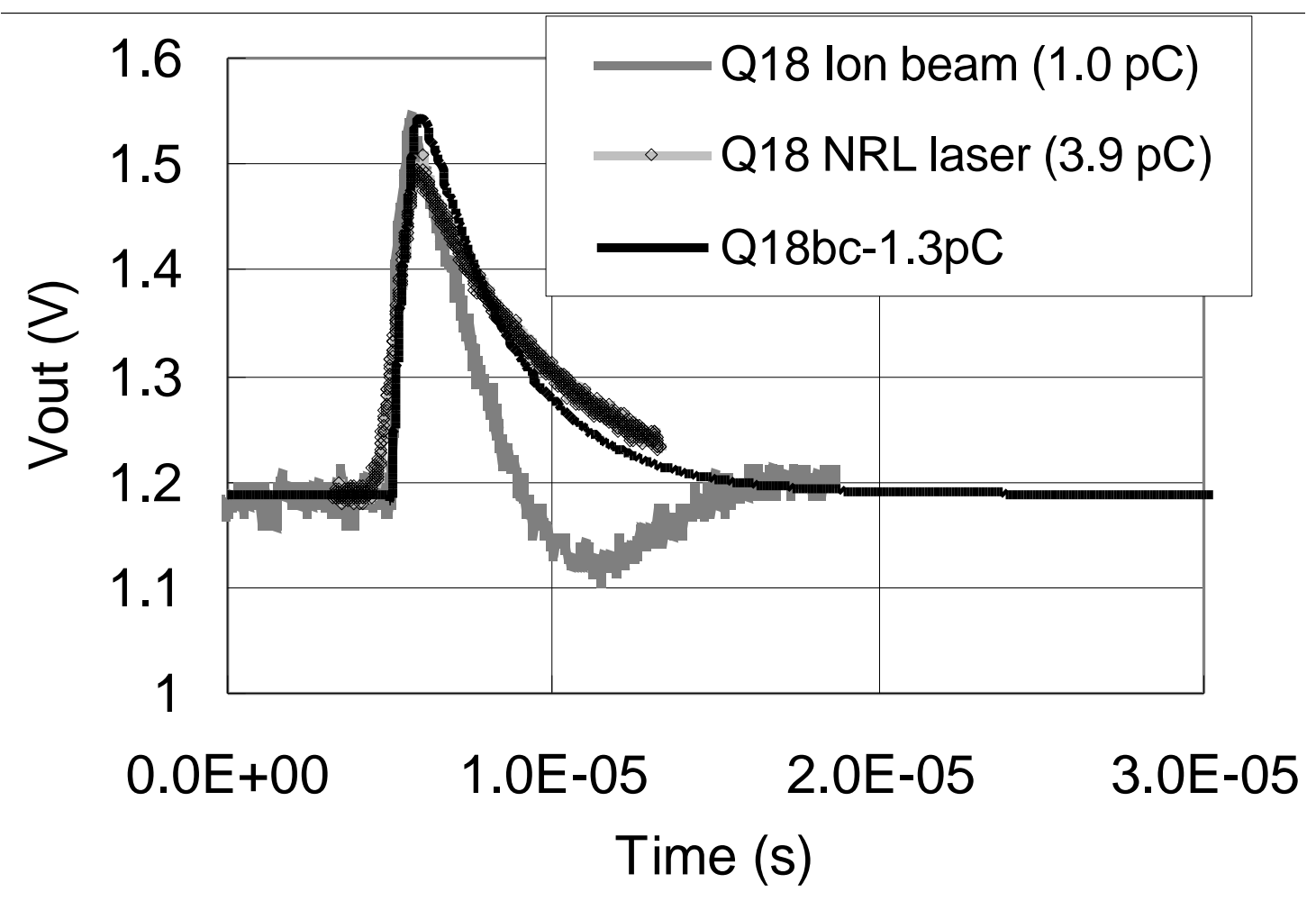




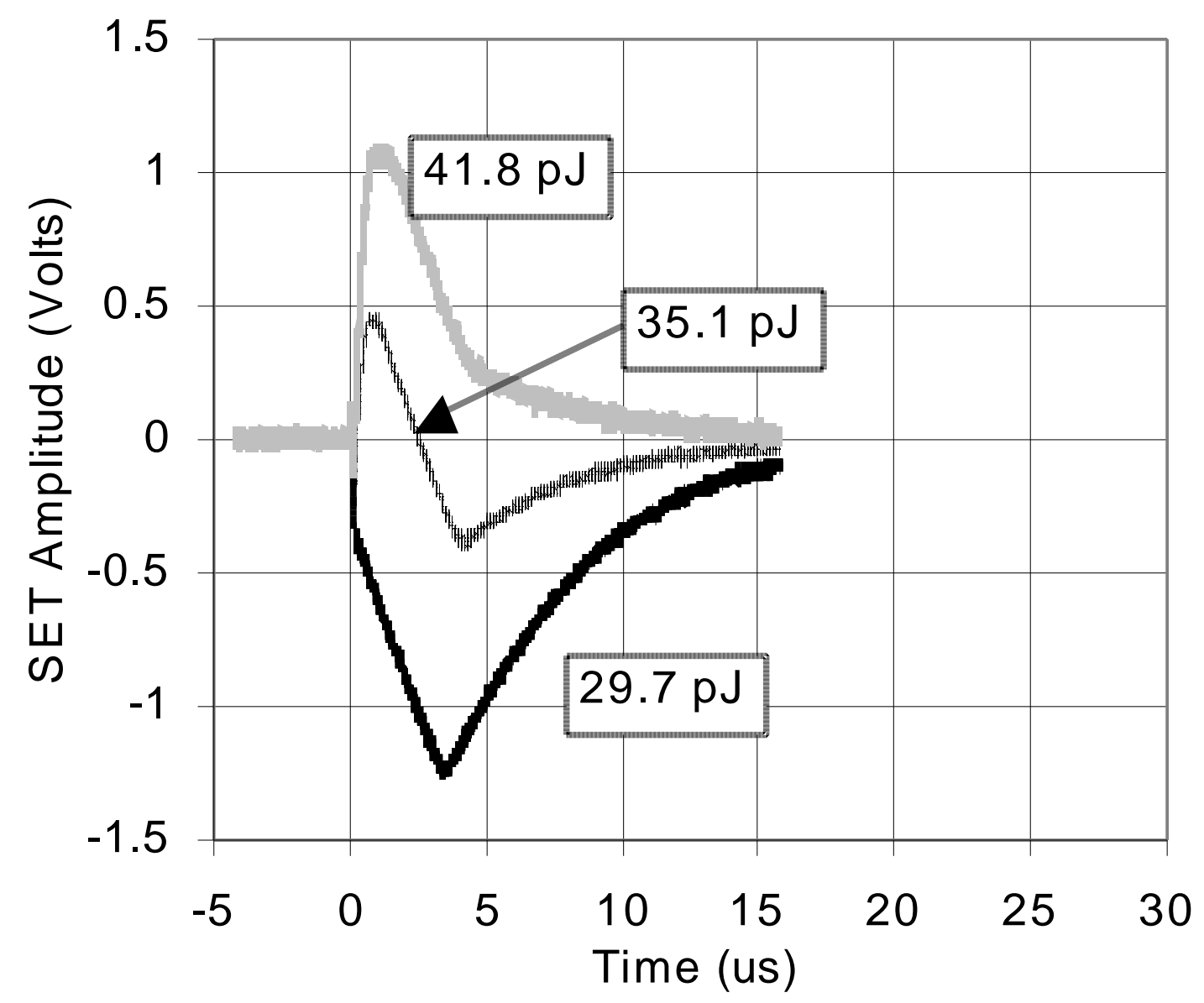




\begin{tabular}{ccc}
\hline Transistor & $\begin{array}{c}\text { Simulated Charge } \\
(\mathrm{pC})\end{array}$ & Laser Charge $(\mathrm{pC})$ \\
\hline Q2bc & 1.8 & 1.3 \\
Q3ce & 1.4 & 0.9 \\
Q4bc & 1.1 & 1.0 \\
Q5bc & 1.6 & 0.9 \\
Q6bc & 1.35 & 2.0 \\
Q9bc & 3.5 & 1.4 \\
Q18bc & 1.3 & 3.2 \\
Q19C5bc & 3.0 & 1.8 \\
"R1"bc & 1.0 & 0.5 \\
\hline
\end{tabular}




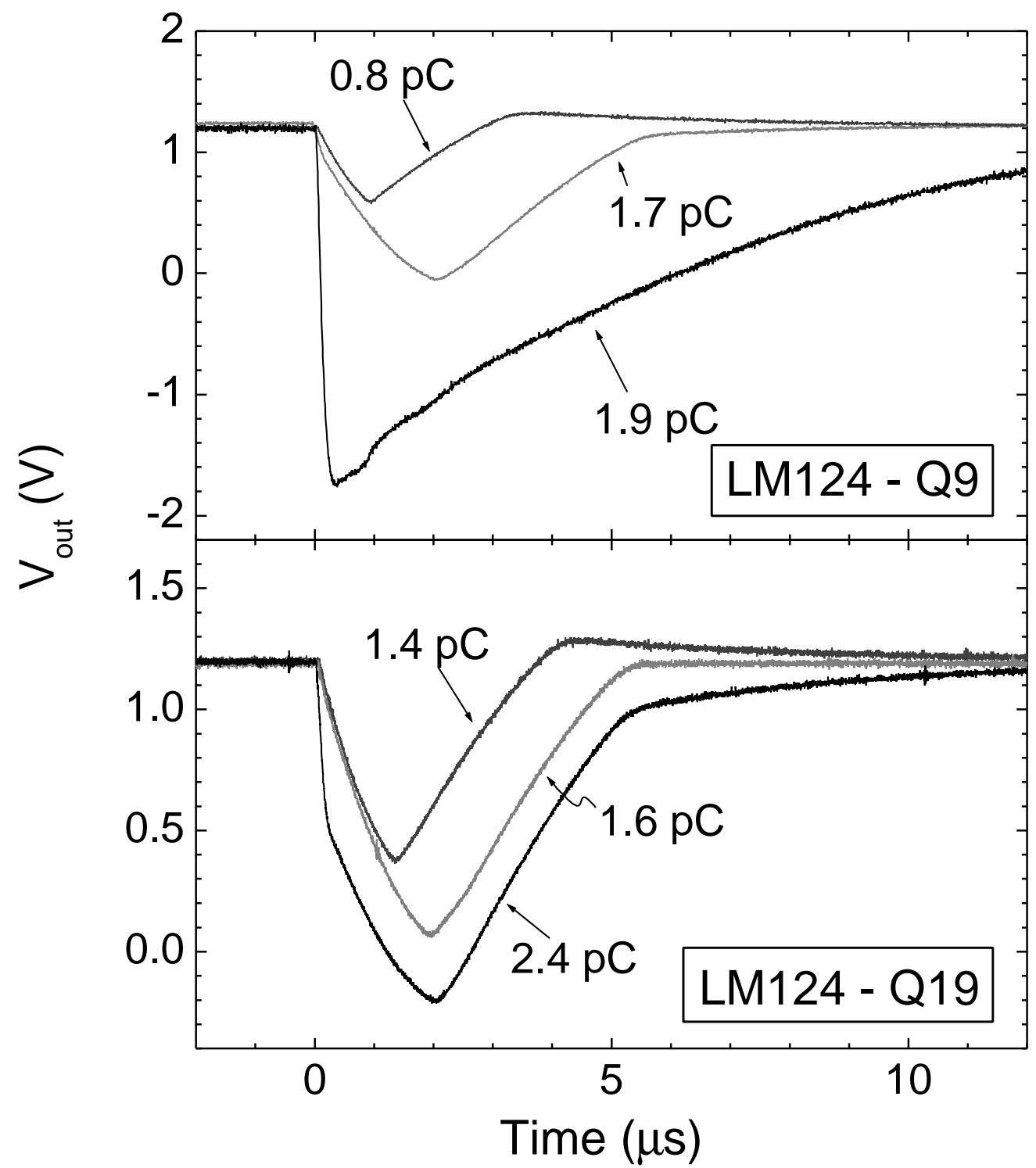

